Aus der kgl. Universitäts-Poliklinik für Haut- und Geschlechtskrankheiten in Berlin (Direktor: Geh. Med.-Rat Prof. Dr. E. Lesser).

\title{
Über einen Fall von Pityriasis rubra (Hebra).
}

\author{
Von \\ Dr. August Halle, \\ Assistenten der Poliklinik. \\ (Hiezu Taf. X.)
}

Bei der großen Seltenheit der zuerst von $\mathrm{F}$. von $\mathrm{Hebra}$ als besonderer Typus beschriebenen und als Pityriasis rubra scharf charakterisierten Krankheit darf wohl jeder neue zur Beobachtung kommende Fall auf einiges Interesse rechnen. Ehe ich an die Beschreibung des Krankheitsfalles herantrete, den mein Chef, Herr Geh.-Rat L e ss e r, mir gütigst zur Veröffentlichung überließ, sei es mir gestattet, in aller Kürze einige geschichtliche Daten zu bringen, welche darlegen sollen, daß der Begriff der Pityriasis rubra trotz der Schilderung $\mathrm{Hebras}$ keiner einheitlichen Auffassung begegnete und da $\beta$ besonders im Auslande noch heute die Neigung besteht, diesen Begriff zu verallgemeinern und die Pityriasis rubra nicht als Krankheit sui generis anzusehen.

Schon vor Hebra haben ältere Autoren wie Willan (1), Batemann (2), Alibert (3), Devergie (4), Fuchs (5) u. a. mit der Bezeichnung Pityr. rubra Krankheitsbilder belegt, die wohl nur zum kleinsten Teil dem $\mathrm{Heb}$ ra schen Typus hinzuzurechnen wären, in der Mehrzahl aber sicher nur generalisierte Ekzeme, exfoliative Dermatitiden und andere Dermatosen betreffen. Hebra (6) wollte mit dem Namen P. r. lediglich eine ganz bestimmte Krankheit bezeichnen, „welche während ihres ganzen Verlaufes von keiner anderen Erscheinung begleitet 
wird als von einer andauernden intensiv dunkelroten Färbung der Haut ohne bedeutende Infiltration, ohne Knötchenbildung, ohne Entwicklung von Schrunden, ohne Nässen oder Bläschenbildung, welche mit geringem Jucken verbunden ist und selten auf einzelne Hautstellen lokalisiert erscheint, sondern meist die ganze allgemeine Decke zu ihrem Sitze erwählt".

Dieser Symptomenkomplex ist dann später von einzelnen deutschen Autoren - besonders von solchen der Wiener Schule - erweitert und ergänzt worden. So erwähnt Hans von $\mathrm{He}$ bra (7) die - freilich schon im Namen "Kleienflechte" ausgedrückte - verschieden starke Desquamation der Epidermis und betont die Unheilbarkeit des Leidens.

Kaposi (8) sah u. a. "vielfache Einrisse in der Epidermis, auch Loshebung derselben auf große Strecken und decubitusähnliche Geschwüre“. Die Prognose stellt er als nicht absolut ungünstig hin.

Schwimmer (9) betont ebenfalls, daß es neben schwereren auch leichtere Formen der Krankheit gebe.

Im Gegensatz zu den deutschen Autoren, die bis in die neuere Zeit hinein in der Hauptsache stets an $\mathrm{Hebras}$ erster Schilderung festhielten, neigt man in England und Amerika bis auf den heutigen Tag dazu, alle mit allgemeiner Rötung und Schuppung einhergehenden Krankheiten einschließlich der Pityriasis rubra dem Vorschlage Wilsons (10) entsprechend als "Dermatitis exfoliativa" zu bezeichnen, oder auch den Namen "Pityriasis rubra" auf generalisierte Ekzeme und andere Dermatosen anzuwenden. In Frankreich hat vor allem Brocq (11) durch seine ausgezeichneten Arbeiten sehr viel zur Klärung der Verhältnisse beigetragen. Er stellt zwar eine Anzahl anderer Krankheitsbilder $\mathrm{n}$ eben die Pityriasis rubra, erkennt aber die Selbständigkeit der letzteren an. Er unterscheidet:

1. Erythème scarlatiniforme desquamatif on dermatite exfoliative aiguë benigne.

2. Dermatite exfoliative généralisée proprement dite ou subaiguë.

3. Dermatite exfoliative généralisée chronique.

4. Pityriasis rubra grave de $\mathrm{Hebra}$.

5. Pityriasis rubra subaiguë bénin. 
Auf ausführlichere Angaben über die Wandlungen, die der Begriff der Pityriasis rubra im Laufe der Jahre in den verschiedenen Ländern und von seiten einzelner Autoren erfahren hat, glauben wir verzichten zu können, da J a das s o hn (12) bereits einen historischen Überblick über diesen Punkt gegeben hat, der an Ausführlichkeit nicht zu übertreffen ist. Auch bezüglich der Literatur bis zum Jahre 1892 möchte ich auf die erschöpfenden Angaben dieses Autors verweisen.

Ich gehe jetzt zur Schilderung des von mir beobachteten Krankheitsfalles über und gebe zunächst die Krankengeschichte, um im Anschlusse daran einzelne Symptome besonders zu besprechen.

V. G., Lokomotivführer, 36 Jahre alt, kam am 19. September 190 t in die Behandlung der Poliklinik.

Anamnese: Patient kann sich keiner schweren Krankheit entsinnen. Das jetzige Leiden besteht seit 6-7 Jahren. Es begann am linken Oberschenkel angeblich in Form kleiner Bläschen auf gerötetem Grunde. Es war anfangs ein Fleck von Markstückgröße, welcher stark juckte und näßte. Während sich dieser erste Herd allmählich vergrößerte, erschienen neue Flecke am 1. Unterarm und etwas später am Penis mit gieichzeitiger Anschwellung der Inguinaldrüsen. Diese Stellen wurden mehrere Wochen von einem Arzt mit gelbem Streupulvex (Dermatol?) behandelt. Später trat dann am r. Oberschenkel ein roter Fleck auf, der sich ebenfalls allmählich ausbreitete, ohne Knötchen und Bläschen zu zeigen; allmählich trat an diesen Stellen Schuppung ein. Ebenso besteht seit 4-5 Jahren ein Herd auf der 1. Seite des Unterleibes. Im Laufe der letzten 2 Jahre breitete sich die Affektion auf beide Unterschenkel und zuletzt auf den rechten Arm aus, indem vorzugsweise die Beugefächen befallen wurden. Auffallend war dem Patienten selbst das Freibleiben der Gelenke.

Im November 1903 erlitt Patient einen Eisenbahnunfall (Zusammenstoß) und leidet seitdem an nervösen Beschwerden (Herzklopfen, Kopfund Rückenschmerzen, innere Unruhe).

Die Behandlung bei verschiedenen Ärzten bestand bisher (seit 1897) in der Anwendung von Bädern, Salben und Streupulver; auch eine Schachtel Arsenpillen bat Patient genommen, ohne die geringste Besserung zu bemerken. Der Krankheitsprozel schritt immer weiter vorwärts und peinigte den Patienten durch den häufig sehr starken Juckreiz.

Am 19./IX. 1904 kam Patient in die Behandlung der Poliklinik. An beiden Armen fiel ein stark ekzematöser Reizzustand zunächst ins Auge. Dieses durch Packungen und Bäder in einer Nervenheilanstalt hervorgerufene Ekzem wurde zunächst behandelt, worauf das in folgendem geschilderte Krankheitsbild klar hervortrat, 
26./I. 1905. Status praesens: Mittelgroßer, mittelkräftiger Mann von $65 \mathrm{~kg}$ Körpergewicht, der in den letzten $11 / 2$ Jahren $12 \mathrm{~kg}$ abgenommen hat. Muskulatur leidlich entwickelt. Fettpolster mittelstark. Gesichtsfarbe etwas bläulichrot. Appetit gut.

Die Körperhant ist an den Extremitäten, aber auch an den großen Flächen des Rumpfes in sehr eigenartiger Weise gerötet und zwar so, daß speziell an den abhängigen Teilen eine livide Verfärbung vorherrscht, während am Rumpf die Farbe etwas frischer ist, aber auch eine bläuliche Nuance hat. Auf Druck verschwindet die Färbung und kehrt nur auffallend langsam zurück (in ca. $1 / 2-1$ Minute). An den erkrankten Partien findet sich überall eine Schuppung in feinen kleinen Lamellen, so dal beim Entkleiden eine größere Menge Schuppen zu Boden fällt. Die Schuppen sind dünn, nur an ganz wenigen Stellen linsengroß und darüber und hängen mitunter nur noch an einer Seite fest.

Die Lokalisation der Erkrankung ist folgende: Bis auf die Fußränder, Sohlen, Zehen, Fersen, Kniekehlen und einem 4 Finger breiten Streifen in jeder Leistenbeuge sind die unteren Extremitäten völlig von der Krankbeit ergriffen. An den oberen Extremitäten sind die Hände nur im Bereich des Handrückens, die Unter- und Oberarme fast vollständig befallen, während die Ellenbeugen ziemlich frei sind. Am Skrotum - besonders rechts - findet sich eine bläulichrote Färbung, aber keine deutliche Schuppung. Die Rumpfhaut ist am wenigsten betroffen und zwar nur die Mittelinie des Rückens bis zur oberen Kreuzbeingegend und die Schulterblätter. Hals und Brust sind bis anf kleine Zonen frei. Aber auch hier bemerkt man streckenweise als Vorläufer der Erythrodermie eine netzartige Cutis marmorata-ähnliche Verfärbung und deutliche Schuppung.

Untersucht man die erkrankte Haut genauer, so bemerkt man an den älteren Stellen (Oberschenkel) eine deutliche Atrophie, welche sich auch in dem sehr spärlichen Vorhandensein von Follikeln und Lanugohaaren kundgibt. Die Haut läßt sich hier zigarettenpapierartig zusammenschieben, zeigt ein mäßiges gelbliches Infiltrat, ist aber in übrigen weich und dünn. An den frischeren Stellen ist infolge der Infiltration das Leistennetz verbreitert. An manchen Stellen treten infolge der Atrophie die Venen ein wenig hervor. Salbt sich Patient nicht regelmäßig ein, so tritt starke Schuppung ein.

Die Lymphdrüsen sind in beiden Leisten deutlich geschwollen (ein Kranz von Bohnen- bis Haselnußgröße). Femoraldrüsen walnußgroß, Zervikaldrüsen böchstens erbsengro $B$, Submental- und Submaxillardrüsen knapp linsengroß, Supraclaviculardrüsen linsengroß, Cubital- und Paramamillardrüsen nicht deutlich, Axillardrüsen bis gut bohnengroß.

Sämtliche Drüsen sind nicht schmerzhaft. Die Hautvenen an den Armen treten deutlich hervor.

Patient gibt an, daß wenn er schwitzt, die stärker erkrankten Teile keine Schweißabsonderung zeigen. Wenn Patient sich entblößt, tritt sofort Frostgefühl ein. 
Haare and Nägel weisen keine Besonderheiten auf.

Am Nervensystem wurden außer gesteigerter Reflexerregbarkeit keine Besonderheiten festgestellt.

Blutbefund :

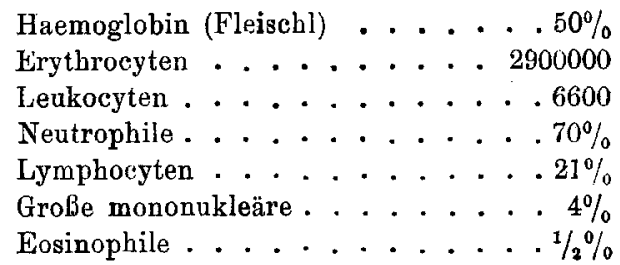

Über der l. Lungenspitze findet sich eine Dämpfung, über beiden Lungenspitzen katarrhalische Geräusche.

Der Urin ist frei von Albumen und Saccharum.

26./I. 1905. Etwas oberhalb der Mitte des 1. Oberschenkels, wo die Atrophie am stärksten ausgeprägt ist, wird eine Excision gemacht.

3./II. 1905. Excision an der Innenseite des l. Oberarms 4 Finger breit unter der Achselfalte zwecks histologischer Untersuchung.

Diagnose: Pityriasis rubra.

7./II. 1905. Patient erhält Arsenpillen à 0.005.

15./II. 1905. Die Arsenpillen werden gut vertragen; gegen den Juckreiz 1\% Thymolspiritus und Puder.

21./II. 1905. Da der Thymolspiritus nicht vertragen wird, soll Patient sich nur einpudern.

3./III. 1905. Patient hat bisher 100 Arsenpillen genommen. Der starke Juckreiz hält an. Weitere 100 Pillen. Gegen die dem Patienten lästige Schuppung täglich mehrmaliges Einfetten mit Borlanolin-Vaseline.

9./III. 1905. Durch das Einfetten der Haut wird die Schuppung auf ein Minimum beschränkt; der Juckreiz besteht fort.

17./III. 1905. Da die Arsentherapie weder auf die Schuppung noch auf den Juckreiz einen merklichen Einfluß ausübt, soll Patient allmählich damit aufhören.

1./IV. 1905. Patient erhält 100 Karbolpillen à 0.005 , von denen er zunächst mittags eine nehmen soll.

10./IV. 1905. Die Karbolpillen werden gut vertragen. Der Urin ist frei von Albumen. Der Juckreiz läßt nach.

17./IV. 1905. Das Jucken hat ganz aufgehört. Alb.

26./IV. 1905. Patient hat bisher 100 Karbolpillen genommen, da sie dauernd gut vertragen werden, Fortsetzung. In der Beschaffenheit der Haut ist bisher keine Änderung eingetreten, auch hat der Krankheitsprozeß die im Januar festgestellten Grenzen bisher nirgends überschritten.

9./V. 1905. Bis heute hat Patient 200 Karbolpillen genommen. Bei regelmäBiger Prüfung wird der Urin stets frei von Eiweiß gefunden.

An wärmeren Tagen erscheinen dem Patienten die erkrankten Hautpartien blasser als bei kalter Witterung. Über den Augenlidern zeigt sich eine leichte Rötung und Schuppung. 
16./V. 1905. Die Rötung und Schuppung über den Augenlidern ist wieder völlig verschwunden.

22./V. 1905. Stat. idem.

31./V. 1905. Patient hat bisher 300 Larbolpillen genommen, Fortsetzung. Der Juckreiz ist bisher nie wieder aufgetreten, die Schuppung besteht fort, wenn Patient sich nicht einsalbt.

6./VI. 1905. Die wärmere Witterung vermindert die subjektiven Beschwerden des Kranken; das Spannungsgefühl ist geringer. Auch objektiv läßt sich ein deutliches Abblassen einiger Hautbezirke erkennen. Fortsetzung der bisherigen Therapie.

30./VI. 1905. In den letzten Wochen ist keine deutliche Veränderung im Krankheitsbilde erkennbar geworden, nur hat es den Anschein, als ob die bisher Cutis marmorata-ähnliche Rötung der Handrücken einem mehr diffusen düsteren Rot Platz mache, indem die hellen Stellen durch die Konfluenz der roten Flecke immer kleiner werden; nirgends aber ist ein Übergreifen des Krankheitsprozesses über die im Januar konstatierten Grenzen bemerkbar. - Das Allgemeinbefinden ist schwankend, doch ist die Mehrzahl der Beschwerden auf die traumatische Neurose des Kranken zurückzuführen. Die lästigsten Symptome des Hautleidens sind auch heute noch die besonders bei kühlerem Wetter fühlbare Spannung in den am meisten veränderten Partien, sowie das Kältegefühl beim Entkleiden.

Der Juckreiz ist dauernd verschwunden.

Das Nahrungsbedürfnis ist ein auffallend großes.

Patient geht auf 6 Wochen in die Sommerfrische und soll sich nach seiner Rückkehr wieder vorstellen. Er hat bisher 600 Karbolpillen genommen, die dauernd gut vertragen werden.

4./VIII. 1905. Der Patient ist von der Reise zurückgekehrt und macht mit seinem gebräunten Gesicht, dem lebhaften Ausdruck seiner Augen einen günstigen Eindruck. Die erkrankten Hautpartien zeigen in keiner Weise eine wesentliche Veränderung. Das Allgemeinbefinden ist besser als vor der Abreise.

25./VIII. 1905. Die schon im Juni wahrgenommene Zunahme der Rötung auf den Handrücken beginnt ganz allmählich dentlicher zu werden. An diesen Stellen erscheint die Hant jetzt leicht infiltriert und weist eine mehr livide Verfärbung auf, die nach Druck nur langsam zurückkehrt.

11./IX. 1905. Keine wesentliche Veränderung.

4./X. 1905. Bis heute hat Patient 800 Karbolpillen genommen. Alb. Seit Eintritt kühlerer Witterung fühlt sich der Patient wieder auffallend unbehaglich.

10./I. 1906. Bei der zur Zeit herrschenden kalten Witterung zeigen die älteren Krankheitsherde auch nach längerem Verweilen im warmen Raume ein auffallend düsteres Rot. Im allgemeinen hat der Prozeß die vor einem Jahre festgestellten Grenzen nirgends erheblich überschritten; nur die Haut der beiden Handrücken hat sich auffallend verschlechtert. 
Die blaurote Verfärbung hat sich diffus bis zum Gelenk der I. und II. Phalange sämtlicher Finger ausgedehnt.

Wegen Verdauungsstörungen hat Patient seit Anfang des Jahres keine Pillen mehr genommen. Da der Juckreiz dauernd verschwunden bleibt, soll die Therapie vorläufig versuchsweise nur in warmen Bädern und in häufigen Einreibungen mit Bor-Lanolin-Vaseline bestehen.

Körpergewicht $66,2 \mathrm{~kg}$.

5./II. 1906. Das Allgemeinbefinden ist besser als im Januar. Die Haut hat sich seitdem nicht wesentlich verändert. Um das dem Patienten aus äußeren Gründen sehr lästige Fortschreiten des Krankheitsprozesses auf den Handrücken nach Möglichkeit einzuschränken, sollen jetzt Wechselbäder für die Hände und nachfolgende Einreibungen mit $5 \%$ Tanninglyzerin versucht werden.

20./Ir. 1906. Die Wechselbäder werden vom Patienten sebr angenehm empfunden und sollen daher fortgesetzt werden.

5./IV. 1906. Stat, idem.

1./V. 1906. Seit Eintritt der wärmeren Witterung fühlt sich Pat. wieder viel wohler. Die Haut hat sich nur wenig im ungünstigen Sinne verändert. Im großen und ganzen hat aber die frühere mehr netzförmige Rötung ïber den Schulterblättern einem mehr diffusen Rot Platz gemacht; auch schuppen diese Stellen stärker als zuvor. Körpergewicht $67 \cdot 2 \mathrm{~kg}$.

25./V. 1906. Stat. idem. Patient geht in die Sommerfrische.

2./VIII. 1906. Patient ist aus der Sommerfrische zurückgekehrt. Er ist mit seinem Allgemeinbefinden zufrieden. Die Haut zeigt dieselbe Beschaffenheit wie im Mai.

1./IX. 1906. An der linken Halsseite hat sich ein neuer etwa markstückgroßer Krankheitsherd gebildet.

4./X. 1906. Die kältere Witterung verursacht dem Patienten wieder viel Unbehagen. - Der älteste atrophische Herd am linken Oberschenkel zeigt einige Rhagaden. Patient soll diese Stellen besonders sorgfältig einfetten.

28./X. 1906. Der Zustand der Haut des Handrückens hat sich in den letzten Wochen sehr verschlechtert. Die Haut ist bläulichrot, glänzend und gespannt. Patient soll wieder Wechselbäder gebrauchen.

15./XI. 1906. Stat. idem.

20./XII. 1906. Der im September zuerst wahrgenommene neue Krankheitsherd am Halse hat sich etwas vergrößert.

13./II. 1907. Während der strengen Kälte hat sich Patient sehr unbehaglich gefühlt. Beim Entkleiden fallen wieder viele Schuppen zu Boden. Die Haut des linken Oberschenkels zeigt hochgradige Spannung und Neigung zur Rhagadenbildung.

Aus dem oben mitgeteilten Status vom 26. Januar 1905 ergibt sich, daß damals der Patient - ebensowenig wie in der Folgezeit - Erscheinungen bot, die zu der Vermutung hätten Anlaß geben können, daß die ausgedehnten Hautveränderungen 
sekundären Charakters seien. Während der ganzen Beobachtungszeit, die also rom genannten Tage $a b$ bis beute eine Frist von über 2 Jabren umfaßt, zeigten sich niemals Knötchen oder Bläschen; der Krankheitsprozeß behielt vielmehr auch während seines überaus langsamen aber unaufbaltsamen Weiterschreitens stets den Charakter einer primären Erythrodermie. Der sehr intelligente Patient gibt selbst an, daß die Ausbreitung seines Leidens nie wit Knötchenbildung oder Nässen rerbunden war. Nur der erste Kranheitsherd am linken Oberschenkel soll anfangs Bläschen auf gerötetem Grunde gezeigt haben. Nach dem ganzen Krankheitsverlaufe sind wir aber vollauf berechtigt diese Erscheinung als zufällig anzusehen, ebenso wie das Ekzem, an welchem der Patient bei einem seiner ersten Besuche in der Universitäts-Poliklinik litt. Es war durch hydro-therapeutische Maßnahmen hevorgerufen und verschwand sehr bald, nachdem sich der Patient in unsere Behandlung begeben hatte. Auf Grund unserer Beobachtungen und der klaren Anamnese konnten wir demnach von vornherein alle sekundären Erythrodermien ausschließen, also alle jene Fälle, die Bazin (13) als Herpétides exfoliatrices bezeichnet und die nichts anderes darstellen als generalisierte Fälle ron Ekzem, Psoriasis, Pemphigus und anderen Dermatosen. Von den primären Erythrodermien konnten wir auf Grund des Krankheitsverlaufes alle jene Formen unberücksichtigt lassen, welche als Prodrome anderer Affektionen z. B. der Mycosis fungoides auftreten. Auch die mit Leukämie und Pseudoleukämie einhergehenden Erythrodermien kamen wegen des normalen Blutbefundes nicht in Betracht. Schließlich hatten wir unter den allein zu berücksichtigenden primären chronischen Erythrodermien noch an die Dermatitis exfoliativa (WilsonBrocq) zu denken, doch ist dieses Leiden auf eine viel kürzere Zeit zusammengedrängt und meist schon innerhalb eines Jahres zyklisch abgelaufen. Auch erfolgt die Schuppung bei der Dermatitis exfoliativa in viel größeren Lamellen, die Haupthaare und Nägel fallen aus, während in unserem Falle die Nägel völlig intakt blieben. Von den chronischen Erythrodermien kam schließlich nur noch die Pityriasis rubra (Hebra) in Betracht, und wir stellten diese Diagnose auch mit Berück- 
sichtigung des histologischen Befundes, von welchem weiter unten die Rede sein soll.

Um nicht Bekanntes und Nebensächliches zu wiederholen, will ich jetzt nur noch an die markantesten Erscheinungen unseres Falles einige Betrachtungen knïpfen.

Die schon von Hebra als Hauptsymptome der Krankheit hervorgehobene Rötung und Schuppung bot keine wesentlichen Abweichungen von den in der Literatur beschriebenen einwandfreien Fällen. Das Fortschreiten der Rötung erfolgte in der Weise, daß sich an die Grenze der schon befallenen Hautpartien eine Cutis marmorata-ähnliche Zeichnung einstellte, in welcher allmählich - oft erst im Verlauf von Monaten - die normalen Hautpartien immer kleiner wurden, bis die neu erkrankten Stellen dieselbe diffuse Rötung zeigten wie die alten Herde.

Die Schuppung war auch in unserem Falle kleinlamellös und erfolgte ununterbrochen, wenn der Patient sich nicht mehrmals am Tage einsalbte. Beim Entkleiden des Patienten erschien der Boden wie mit Mehl bestreut.

Von den subjektiven Beschwerden war dem Patienten neben dem Juckreiz und dem Gefühl von Spannung in den schon atrophischen Hautpartien das Kältegefühl am lästigsten. Diese auch bei anderen ausgebreiteten Hautaffektionen beobachtete Erscheinung ist zweifellos in unserem Falle auf die gestörte Wärmeregulation des Körpers zuruckzuführen, die hauptsächlich in der mangelhaften Funktion der Hautgefäße sowie in dem allmählichen Schwund der kontraktilen Eiemente und Drüsen ihren Grund hat. Die auch histologisch bestätigte Beeinträchtigung des Gefäßapparates gab sich zunächst kund in der auffallend lange persistierenden Anämie, wenn man durch kurz dauernden Finger- oder Spateldruck einzelne Partien der jüngeren Krankheitsherde blutleer gemacht hatte. An den älteren schon in Atrophie übergegangenen Herden ließ sich dies Phänomen nicht mehr so deutlich nachweisen. Am auffallendsten aber äußerte sich die Störung der Hautzirkulation bei Witterungswechsel. Selbst im Sommer reagierte der Patient auf von Gesunden kaum wahrgenommene Temperaturschwankungen in der empfindlichsten Weise. An kühleren Tagen war das Frostgefühl in einer Weise gesteigert, die zu dem Temperaturabfall 
in gar keinem Verhältnis stand. Den Verlust an Kalorien, den die Körperwärme durch die mangelhafte Funktion des wichtigsten Wärmeregulators, der Haut, erleidet, hat der Organismus bis zum heutigen Tage auffallend gut ertragen. Sowohl diesen Wärmeverlust wie auch den Verlust an Eiweißkörpern, wie er sich beständig durch die enorm gesteigerte und in Schuppung sich äußernde Zellproliferation kundgibt, hat der Patient, wie es scheint, durch einen seit Jahren bestehenden wahren Heißhunger wieder auszugleichen verstanden. Wenn Jadassohn dem Stickstoffverlust durch eine so hochgradige Schuppung, wie sie als eins der Hauptsymptome bei P. r. beobachtet wird, jede Bedeutung für den Stoffwechsel abspricht und zum Vergleich Psoriatiker heranzieht, die trotz ausgedehnter Schuppenbildung in bestem Ernährungszustande bleiben, so übersieht er m. E., daß die oft fest und lange haftenden Schuppen der Psoriasis lediglich eine Verhornungsanomalie darstellen, während die beständige, ununterbrochene Schuppung der P.r. der Ausdruck einer krankhaft gesteigerten Zellproliferation ist (zahlreiche Mitosen!), die auf die Dauer ganz gewiß nicht gleichgültig für den Organismus sein kann. Stoffwechselerkrankungen wie Diabetes $u$. a. haben während des bisherigen Krankheitsverlaufes bei unserem Patienten nie bestanden. Das Körpergewicht entsprach in der Beobachtungszeit stets der Größe und dem Alter des Patienten und wies nie erhebliche Schwankungen auf.

Inwieweit die nervösen Beschwerden auf den oft hochgradigen Juckreiz resp. auf seine Hautaffektion oder auf eine, nach einem Unfall aufgetretene traumatische Neurose zurückzuführen waren, ließ sich anfangs schwer entscheiden. Nachdem durch die Therapie (Karbolpillen) der Juckreiz beseitigt worden ist, glauben wir die weiter fortbestehenden nervösen Beschwerden als eine Folge seines Unfalles ansehen zu dürfen, den er in seiner Tätigkeit als Lokomotivführer vor mehreren Jahren erlitt.

Von den übrigen klinischen Erscheinungen verdienen dann noch die allgemeinen Drüsenschwellungen, die der Patient darbot, eine eingehende Besprechung. 
In Hebras Schilderung der Pityriasis rubra ist ron Anschwellung der Lymphdrüsen noch nicht die Rede. Den ersten Hinweis auf allgemeine Drüsenschwellungen finden wir bei Cahn (14), welcher einen in Würzburg von Rienecker (15) demonstrierten Fall von P. r. beschreibt.

Ferner erwähnen Anschwellung der oberflächlichen Lymphdrüsen: Elsenberg (16), Leviseur (17) und ror allem Jadassohn, welcher bereits im Jahre 1892 diesem Symptom ein besonderes Kapitel widmete und feststellte, daß unter 18 sicheren Fällen von P. r. Tuberkulose der inneren Organe oder der Lyraphdrüsen mit Sicherheit zu konstatieren war. Er selbst fand in zwei Fällen von P. r. in den Lymphdrüsen tuberkulöse Veränderungen sowie Tuberkelbazillen und lenkte somit die Anfmerksamkeit auf die damals noch wenig bekannte primäre Tuberkulose der oberflächlichen Lymphdrüsen, betonte aber, daß "von einem für uns verständlichen gesetzmäßigen inneren Zusammenhange beider Affektionen - der Tuberkulose und der P. r. keine Rede sein könne".

Seit jener Zeit haben sich die bekannt gewordenen Fälle von P. r. um eine Anzahl vermehrt, bei der eine gleichzeitige Tuberkulose teils sicher konstatiert wurde, teils mit Wahrscheinlichkeit angenommen werden konnte.

Bruusgaard (18) fand in einem von ihm als „Erythrodermia exfoliativa universalis tuberculosa" beschriebenen Krankheitsfalle nicht nur in den Lymphdrüsen sondern auch in der erkranlsten Haut selbst Tuberkelbazillen, zu einem Präparat Fingers fand Jadassohn ebenfalls tuberkulöse Veränderungen. Auch in dem ron Kopytowski und Wielowieyski (19) beschriebenen Falle glaubt Török (20) die geschilderten histologischen Veränderungen, auf die wir nochmals zu sprechen kommen werden, auf Tuberkulose zurücifüihren zu müssen. Kanitz (21) hat in neuester Zeit einen Fall von P. r. beschrieben, bei dessen Sektion tuberkulöse Veränderungen in den Lungen sowie die Residuen einer in der Kindheit abgelaufenen Coxitis gefunden wurden.

Endlich verdanke ich einer mündlichen Mitteilung des Herrn Prof. Wolters die Kenntnis, daß sich in seiner Klinik vor einiger Zeit eine Patientin befand, die an P. r. litt und 
an einer ausgedehnten Tuberkulose der Beckenorgane zu Grunde ging.

Zu unserem Falle haben wir Tuberkulose mit Sicherheit bisher nicht feststellen können, jedoch ist die erwähnte Dämpfung an der linken Lungenspitze kaum anders zu deuten als durch die Annahme eines alten, anscheinend völlig ausgeheilten, tuberkulösen Herdes. Von einer Tuberkulinreaktion zu diagnostischen Zwecken mußten wir wegen der hochgradigen Nervosität des Kranken Abstand nehmen. Auf Grund der oben erwähnten Beobachtungen und Forschungsresultate $\mathrm{J}$ a d a s s o h n s bin ich der Ansicht, daß die auffälligen Drüsenschwellungen unseres Patienten ebenfalls auf eine latente Tuberkulose der Lymphdrüsen zu beziehen sind. Auch die Tatsache, daß der Patient hereditär belastet ist sowie der normale Blutbefund, welcher leukämische und pseudoleukämische Drüsentumoren ausschließt, spricht für diese Auffassung.

Ebe ich das Ergebnis der histologischen Untersuchung unseres Falles schildere, will ich in Kürze das bisher in der Literatur über diesen Punkt bekannt Gewordene zusammenfassen. Von vornherein sei gesagt, daß die Untersuchungsresultate der meisten Autoren im wesentlichen nicht von einander abweichen; geringe Differenzen sind oft dadurch zu erklären, daß den Untersuchern verschiedene Stadien des Krankheitsprozesses zu Gesicht kamen.

Ich berücksichtige nur solche Publikationen, aus denen deutlich hervorgeht, daß es sich um sichere Fälle von $P$. r. im Sinne Hebras handelte.

Die erste ausführliche Schilderung der Gewebsveränderungen bei P. r. verdanken wir Hans $\mathrm{Hebra}$; von späteren Autoren sind vor allem zu nennen: Cahn, Elsenberg, Tommasoli (22), Petrini (23), Jadassohn, Doutrelepont (24), Sellei (25), Kopytowski, Wielowieyski, Tschlenow (26) und Kanitz.

Im Frühstadium fanden H. Hebra, Elsenberg u. a. die Hornschicht verdickt; Kanitz gibt an, daß sie in seinen Präparaten stellenweise das Dreifache der normalen Dicke hatte und fand, wie schon ror ihm $\mathrm{Cahn}$, Petrini, Jadassohn und Doutrelepont, in derselben gut gefärbte Kerne. 
Das Rete Malpighi wurde im frühen Stadium von H. Hebra, Cahn, Elsenberg und Jadassohn als normal bezeichnet, während Petrini und Kanitz dasselbe verdickt fanden. Letztgenannter Autor gibt ebenso wie Doutrelepont an, daß die interpapillären Epithelzapfen verlängert waren. Auffallend viele Kernteilungsfiguren fanden $\mathrm{Jadassohn}$, Doutrelepont und Kanitz.

Petrini, Jadassohn und Doutrelepont erwähnen reichliche in das Rete Malpighi einwandernde Leukocyten.

Sehr auffallend war Jadassohn die - im Gegensatz zum Pigmentgehalt der Cutis - außerodentlich geringe Pigmentierung der Palisadenzellenschicht.

Während im übrigen die Mehrzahl der Autoren darin übereinstimmt, daß die Papillen im Frühstadium normale Form haben und da! die papillären und subpapillären Gefäße erweitert sind, fand Petrini die Papillen verschmälert und die Gefäße sklerosiert. Alle Autoren fanden einen mäßigen Grad von Rundzelleninfiltration und nur in der Nähe der Gefäße größere Zellanhäufungen. Jadassohn und Doutrelepont erwähnen eine auffallende Zahl von Mast- und Pigmentzellen.

Von einer mäßigen Vermehrung der fixen Bindegewebszellen abgesehen wurden die tieferen Cutisschichten stets normal gefunden; nur erwähnen die meisten Autoren das Vorhandensein zahlreicher Pigmentanhäufungen. Entsprechen somit die histologischen Bilder des Frühstadiums der P. r. in der Hauptsache einer mit gesteigerter Epithelproliferation einhergehenden Entzündung, so daß derartige Krankheitsfälle auch mikroskopisch von der Dermatitis exfoliativa subacuta, einer stets zur restitutio ad integrum führenden Krankheit, sich nicht unterscheiden lassen, so ergibt anderseits die Untersuchung des atrophischen Stadiums recht charakteristische Befunde:

Das Epithel, besonders das Rete Malpighi, wird atrophisch, die Papillen flachen ab oder verschwinden vollständig, und die drüsigen Organe der Haut gehen zu Grunde. $\mathrm{Zu}$ diesen Grundzügen stimmen die Schilderungen von H. Hebra, Tommasoli, Jadass ohn, Doutre le pont und Peter (27) überein.

Das subepitheliale Infiltrat enthält nach $\mathrm{Jadassohn}$ in diesem Stadium mehr fixe Bindegewebszellen und Mastzellen. 
Nach Sellei sollen alle diese Veränderungen den in der senilen Haut sich findenden Degenerationsprozessen entsprechen.

Das elastische Gewebe fanden $\mathrm{H}$. Hebra, Cahn und Peter vermehrt, während Jadassohn diese Vermehrung nur für eine scheinbare hält und Kanitz die elastischen Fasern in den Papillen und subpapillaren Schichten beträchtlich vermindert fand.

Petrini beobachtete eine hyaline Degeneration und Obliteration der Gefäße. Auch Tschlenow und Kaposi fanden atheromatöse Veränderungen an den Gefäßen.

Kanitz gibt an, daß die subepithelialen Gefäße erweitert sind und verdickte Wandungen haben.

Außer diesen in der Literatur sich findenden histologischen Untersuchungsresultaten im Früh- und Spätstadium der P. r. verdienen zwei bereits oben kurz erwähnte Einzelbeobachtungen noch eine besondere Besprechung:

Kopytowski and Wielowieyski fanden in ihren Präparaten neben diffuser Entzündung noch circumscripte Herde, welche außer Leukocyten großen Epitheloid- und Pigmentzellen auch typische Riesenzellen enthielten. Außerdem fanden die genannten Autoren in der post mortem excidierten Haut und in einer Inguinaldrüse zahlreiche in Gruppengelagerte Diplokokken, die sich mit Anilinfarben lebhaft färbten, und nahmen an, daß dieser Parasit in direkten Zusammenhang mit der Entstehung der P. r. zu bringen sei.

Als "Erythrodermia exfoliativa universalis tuberculosa" beschreibt $\mathrm{Br}$ u usga ard auch histologisch einen Krankheitsfall, den er zwar nicht als P. $r$. im Sinne $\mathrm{Hebras}$ aufgefaßt wissen will, der aber seiner Ansicht nach sehr viele Berührungspunkte mit dieser Krankheit hat. Histologisch bemerkenswert ist, daß Bru usgaard bei seiner Patientin post mortem eine primäre universelle Lymphdrüsentuberkulose feststellte und auch in einzelnen Hautschnitten typische tuberkulöse Veränderungen sowie Tuberkelbazillen nachweisen konnte.

Ich wende mich jetzt der histologischen Beschreibung unseres Falles zu.

Zur Untersuchung wurde je ein Hautstückchen aus einem frischeren und einem älteren Krankheitsherde excidiert, in 
Alkohol fixiert und in Paraffin eingebettet. In den Schnitten des älteren Krankheitsherdes ist die Horns chi cht verbreitert, stellenweise lamellös aufgeblättert und es finden sich vielfach in ihr deutlich gefärbte Kerne.

Das Epithel ist in allen seinen Lagen ganz beträchtlich verschmälert; streckenweise besteht es nur aus wenigen Zellagen und ist fast überall von massenhaft eindringenden Leukocyten durchsetzt, deren dunkle Kerne sich gegen die auffallend schlecht tingiblen, scheinbar vielfach geschrumpften Kerne der Epithelien scharf abheben.

Das Stratum granulosum besteht streckenweise nur aus einer einzigen Zellreihe mit spärlichem Keratohyalingehalt; hier und da scheint es gänzlich zu fehlen.

Die Zellen des Stratum germinativum sind sowohl in ihrer Gestalt als auch in ihrer Lage zu einander fast überall beträchtlich alteriert durch die das Epithel auseinanderdrängenden Leukocyten. Besonders auffallend ist das Fehlen von Pigment in den Palisadenzellen.

Die Grenze zwischen Epithel und Cutis entbehrt der charakteristischen Zeichnung durch den fast völligen Schwund der Papillen. An vielen Stellen ist diese Grenze überhaupt völlig verwischt, indem die in das Epithel eingewanderten Leukocyten als unmittelbare Ausläufer des subepithelialen Infiltrats erscheinen, welches sich wie ein breites Band zwischen der atrophischen Epidermis und den bis auf geringe Veränderungen normalen tieferen Cutisschichten hinzieht. Dieses Infiltrat besteht aus zahllosen Zellen verschiedenen Charakters. Zum Teil sind es blaßgefärbte Zellen mit ovalem Kern, die als fixe Bindegewebszellen anzusehen sind. Daneben finden sich aber zahlreiche dunkel gefärbte, vorwiegend einkernige Rundzellen und Ehrlich sche Mastzellen. Innerhalb dieses aus so verschiedenartigen Zellen zusammengesetzten Infiltrats sind sämtliche Follikel, Haare und Talgdrüsen zu Grunde gegangen. Elastische Fasern sind nur in den tieferen Schichten des Infiltrats und nur mit starker Vergrößerung in spärlichen Resten wahrzunehmen. In den tieferen Schichten erscheint die Cutis auf den ersten Blick normal, jedoch finden sich bei genauerer Prüfung auch hier auffallende Veränderungen. Im Gegensatz zu 
dem geschilderten diffusen Infiltrat im oberen Teil der Cutis finden sich in der Tiefe nur vereinzelte Infiltrationsherde in der Nähe der Gefäße und Schweißdrüsen. Das elastische Gewebe ist hier wohlerhalten und erscheint nur nach oben $\mathrm{zu}$, an der Grenze des zelligen Infiltrats, etwas verdichtet resp. zusammengedrängt, so daß in mit Orcein gefärbten Präparaten die Grenze zwischen infiltrierten und relativ normaler Cutis durch dichte Züge der dunkel tingierten elastischen Fasern markiert erscheint.

Auffallend verändert sind die Blutg efäße. Sie weisen stellenweise eine eigentümliche Schlängelung auf und ihre Wandung ist verdickt. Am meisten verändert ist die Media, welche überall beträchtlich verbreitert erscheint, viele gut färbbare Kerne enthält und keinerlei Zeichen von Degeneration erkennen läßt.

Die Intima ist in auffallend vielen Gefäßquerschnitten stellenweise abgehoben; wesentliche Veränderungen ließen sich an ihren Zellen nicht nachweisen. Auch die Adventitia bot nirgends bemerkenswerte Befunde.

An Schnitten, welche dem jüngeren Krankheitsherde entstammen, lassen sich die hervorgehobenen histologischen Veränderungen auch sämtlich - wenn auch in geringerem Grade nachweisen. Der einzige Unterschied besteht darin, daß in diesen Präparaten die Epidermis nach Zahl und Anordnung ihrer Zellagen noch relativ normal erscheint, daß sich hier und da noch Reste von Papillen finden und daß das Infiltrat weniger fixe Bindegewebszellen dagegen mehr mononukleäre Rundzellen enthält. Die Verdickung der Gefäßmedia ist auch in diesen Präparaten deutlich nachweisbar.

In den Grundzügen stimmen also die Ergebnise meiner histologischen Untersuchung mit den Angaben der meisten Autoren überein, und ich will in Folgendem versuchen, einige der markantesten klinischen Erscheinungen durch die wahrgenommenen histologischen Veränderungen zu erklären.

Die ununterbrochene Schuppung kommt in den Präparaten durch die Verbreiterung der vielfach lamellös aufgeblätterten Hornschicht, ihrem Reichtum an färbbaren Kernen (Parakeratose), sowie in den zahlreichen Mitosen in der Epidermis zum Ausdruck. Gerade diese von Jadassohn, Doutrelepont 
und Kanitz hervorgehobenen zahlreichen Kernteilungsfiguren beweisen, daß wir es nicht lediglich mit einer Parakeratose, sondern mit einer krankhaft gesteigerten Epithelproliferation $z \mathfrak{u}$ tun haben. Die geschilderten Veränderungen an den Gefäßen - die Verdickung ihrer Media und die dadurch bedingte Rigidität - dürften die Hauptursache der Störung im Wärmehaushalt der Patienten sein, indem die Gefäße die Fähigkeit verlieren, den auf sie wirkenden thermischen oder mechanischen Reizen entsprechend sich zusammenzuziehen oder zu erweitern.

Auch der Mangel sämtlicher Follikel und Drüsen im beschriebenen Infiltrat sowie der Schwund der Mm. arrectores pilorum erklärt uns die Störung der Wärmeökonomie: Gerade die $\mathrm{Mm}$. arrectores pilorum sind es ja, die bei Abkühlung der Außentemperatur durch ihre Kontraktion die sogenannte Cutis anserina hervorrufen und durch die Verdrängung der vorher an der Peripherie befindlichen Blutmengen nach zentralwärts gelegenen Partien einer Herabsetzung der Körpertemperatur vorbeugen, da die nur aus einem Endothelschlauch bestehenden Kapillaren wegen ihres Mangels an konktraktilen Elementen an sich nicht die Fähigkeit besitzen, auf eine Abnahme der Außentemperatur hin sich zusammenzuziehen und somit die in ihnen enthaltene - in ihrer Gesamtheit große - Blutmenge vor Abkühlung zu schützen. Das Fehlen aller dieser Wärmeregulatoren macht es erklärlich, daß die Patienten im unbekleideten Zustande und auch bei geringfügigen Schwankungen der Außentemperatur auffallend leicht frösteln.

Zur Klärung der Ätiologie der Pityriasis rubra habe ich nichts beitragen können, doch sei es mir gestattet in Kürze meine Anschauung über diesen Punkt auszusprechen:

Für das schon von Jadassohn vor mehr als zehn Jahren mit Vermeidung jeglicher Schlußfolgerung hervorgehobene gleichzeitige Vorkommen von Tuberkulose bei an P. r. Erkrankten haben die neueren Publikationen weitere Beispiele gebracht und Jadassohn sowohl wie Török sind der Meinung, daß wahrscheinlich ein Teil der als P. r. beschriebenen Fälle auf Tuberkulose zurückzuführen und vielleicht als Toxituberkulide aufzufassen ist. Mit größerem Nachdruck hat neuerdings K a nitz diese Anschauung auf Grund des von ihm beschriebenen Krank- 
heitsfalles verfochten, indem er gleichzeitig auf die Untersuchungsresultate $\mathrm{Br}$ u u sga ard s hinwies, welcher - wie bereits erwähnt - in einem Falle von universeller Erythrodermio tuberkulöse Veränderungen in den Lymphdrüsen konstatierte. Bei unserem Falle habe ich Tuberkulose nicht mit Sicherheit feststellen, sondern nur als wahrscheinlich annehmen können, so daß ich ibn als beweiskräftig für die erwähnte Anschauung nicht ansehen darf, wohl aber scheint mir der meines Wissens bisher nicht publizierte - Woltersche Fall ein neuer Beweis für den Zusammenhang zwischen P. r. und Tuberkulose $\mathrm{zu}$ sein. Es wird aber weiterer Beobachtungen bedürfen, um die Art dieses Zusammenhangs zu ergründen. Das ist aber nur dann möglich, wenn in Zukunft die Bezeichung "Pityriasis rubra" nicht so verallgemeinert wird wie bisher, sondern nur solche Fälle berücksichtigt werden, die wirklich der Hebraschen Schilderung entsprechen und mit Atrophie endigen. Denn darin liegt $\mathrm{m}$. E. das Wesen dieser chronischen Entzündung, daß sie niemals zu einer restitutio ad integrum sondern stets zur Atrophie führen muß. Bezüglich der vielen in der Literatur als P. $r$. beschriebenen Fälle mit angeblich günstigem Verlauf schreibt $\mathrm{J}$ aris ch (28):

„Sind diese aber als solche von Pityriasis rubra anzusehen? Wir halten diese Frage bei dem heutigen Stande unserer ätiologischen und pathogenetischen Kenntnisse für undiskutierbar und meinen, daß, wenn bei morphologisch mangelhaft ausgestatteten Hautkrankheiten auch die Merkmale des Verlaufes als bedeutungslos hingestellt werden, alle bisherigen Errungenschaften über Bord geworfen werden und ein grenzenloses Chaos platzgreifen mul."

Um Wiederholungen zu vermeiden möchte ich mich über die Prognose des Leidens nur noch mit wenigen Worten äußern. Schon Schwimmer, Besnier (29), Jadassohn u. a. haben darauf hingewiesen, daß es Fälle gibt, die relativ günstig verlaufen. Die Krankheit kann Jahrzehnte hindurch bestehen, schließlich aber gehen die Patienten doch an Marasmus oder Allgemeintuberkulose zu Grunde. Auch in dem von mir beschriebenen Falle besteht die Krankheit schon etwa 10 Jahre, ohne daß die Gesamternährung des Patienten bisher wesentlich 
gelitten hätte. Während der 21/qjährigen Beobachtungszeit traten zu den schon befallenen Hautpartien nur wenige neue Krankheitsherde hinzu, aber jeder neuergriffene Bezirk war ausnahmslos der fortschreitenden Atrophie verfallen, was eine "Heilung“ im Sinne einer restitutio ad integrum ohne weiteres ausschließt. Eine Besserung wurde zuweilen dadurch vorgetäuscht, daß bei wärmerem Wetter das düstere Rot der erkrankten Haut einen mehr gelblichroten Farbenton - die Farbe der jüngsten Krankheitsherde - annahm. Im großen und ganzen gewann ich den Eindruck, daß das Leiden im Winter schnellere Fortschritte machte als wie im Sommer.

Über die T her a p i e bleibt mir wenig zusagen übrig: Keins der bekannten inneren oder äußeren Mittel vermag nach meiner Überzeugung den Krankheitsprozeß aufzuhalten. Die innerliche Darreichung von Karbolsäure, durch welche Kaposi einen rezenten Krankheitsfall geheilt $z u$ haben glaubfe, hat unseren Patienten zwar vom lästigen Juckreiz befreit, auf den Verlauf des Leidens selbst aber sicherlich keinen Einfluß gehabt. Auch das Arsen versagt der P. r. gegenüber.

Äußere Mittel kommen nur in Betracht, soweit sie die subjektiven Beschwerden der Kranken zu lindern und der mit der fortschreitenden Atrophie drohenden Rhagadenbildung vorzubeugen vermögen. $\mathrm{Zu}$ vermeiden sind alle jene Mittel, welche die Trockenheit der Haut steigern könnten; in dieser Hinsicht warnte bereits Kaposi vor dem Gebrauch des Teers. Eine entschiedene Besserung glaubte Leistikow (30) in einem Falle durch einen zweiprozentigen totalen Ichthyoldunstverband erzielt $z u$ haben. In manchen vorgeschrittenen Fällen wird man zum permanenten Wasserbade seine Zuflucht nehmen müssen. Unser Patient empfand warme Bäder sehr angenehm, und das. häufige Einfetten mit Bor-Lanolin-Vaseline hielt die ihm lästige Schuppung in gewissen Grenzen.

Da die meisten - wenn nicht alle - der an P. r. Leidenden auch tuberkulös sind, so ist in jedem Falle auf eine entsprechende Hygiene und günstige Ernährungsverhältnisse Wert zu legen.

\section{Literatur.} mann. 1820 .

1. Willan. Abrégé pratique des maladies de la peau par Bate- 
2. B a t e mann. Praktische Darstellung der Hautkrankheiten, übersetzt von Blasius. Leipzig 1841.

3. Alibert. Monographie des Dermatoses. Paris 1835. II.

4. Devergi e. Traité pratique des maladies de la peau. Paris 1857.

5. Fu ch s. Die krankh. Veränderungen der Haut. Göttingen 1840.

6. Hebra, F. Virchows Handbuch der speziellen Pathologie und Therapie. 1862. III. Bd. 2. H. p. 321.

Idem. Lehrbuch der Hautkrankheiten. I. Aufl. 1862, II. Aufl. 1874. 7. v on Heb ra, H. Lehrbuch der Hantkrankheiten. 1884.

Idem. Pityriasis rubra. Vierteljahrsschrift für Derm. u. Syph. 1876. 2. und 3. Auflage.

8. Kaposi. Pathologie und Therapie der Hautkrankheiten. 1.,

9. Schwimmer. Zur Diagnostik der Pityriasis rubra universalis.

Pester med. Presse 1884.

10. Wilson. Med. times and gazette. 1870.

11. Brocq. Traitement des maladies de la peau. Paris 1890.

12. Jadassohn. Über die Pityriasis rubra (Hebra) und ihre Beziehungen zur Tuberkulose. Arch. f. Derm. u. Syph. 1892.

Idem. Die Tuberkulose der Haut. Mračeks Handbuch. IV. Bd. p. 285.

13. $\mathrm{Baz}$ in. Leçons sur les affections cutan. arthrites et dartreuses.

Paris 1873.

14. Co hn. Über Pityriasis rubra. Dissert. Würzburg 1884.

15. Rienecker. Über Pityriasis rubra. Verhandl. d. Würzburger physikal.-med. Gesellschaft. 1883.

16. Els enberg. Pityriasis rubra universalis. Vierteljahrsschr. für

Derm. u Syph. 1887. p. 727.

17. Levi is e ur. Pityriasis rubra. Journal 1890. XII. p. 492.

18. Bru us ga ard. Beitrag zu den tuberkulösen Hauteruptionen. Erythrodermia exfoliativa universalis tuberculosa. Arch. f. Derm. u. Syph. 1903. Bd. LXVII.

19. Kopitow ski - Wielowicyski. Beitrag zur Klinik und pathologischen Anatomie der Pityriasis rubra Hebrae. Archiv für Dermatol. u. Syph. 1901. Bd. LVII.

20. Török. Die exfoliativen Erythrodermien. Mračeks Handbuch der Hautkrankbeiten.

21. Kanitz. Beitrag zur Klinik, Histologie und Pathogenese der Pityriasis rubra (Hebra). Arch. f. Derm, u. Syph. 1906. Bd. LXXXI.

22. Tommasoli. Beitrag zur Histologie der Pityriasis rubra. Monatsh. f. prakt. Dermat. Bd. IX. 6.

23. Petrini. Congrès international de Derm. Paris 1890.

24. Doutrelepont. Beitrag zur Pityriasis rubra (Hebra). Archiv f. Derm. u. Syph. 1900. Bd. LI.

25. Sellei. Die Pityriasis rubra (Hebra). Arch. f. Derm. u. Syph. 1901. Band LV.

26. Ts ahlenoff. Ein Beitrag zur Kenntnis der Pityriasis rubra.

Arch. f. Dermatol. u. Syph. 1903. Bd. LXIV.

27. Peter. Über Pifyriasís rubra und die Beziehungen zwisehen

Hautkrankbeiten und Pseudoleukämie. Derm. Zeitsohr. 1893-94.

28. J ar i s ch. Die Hautlirankheiten. 1890.

29. Bes nier. Erythrodermies. Traduct. Vol. I. p. 617.

30. Leistikow. Therapie der Hantkrankheiten. 1897.

Die Erklärung der beiden Abbildungen (oberer und unterer Teil eines

Schnittes durch einen älteren Krankheitsherd, Zeiss, 0c. 4, Obj. AA) auf Taf. $X$ ist dem Texte zu entnehmen. 

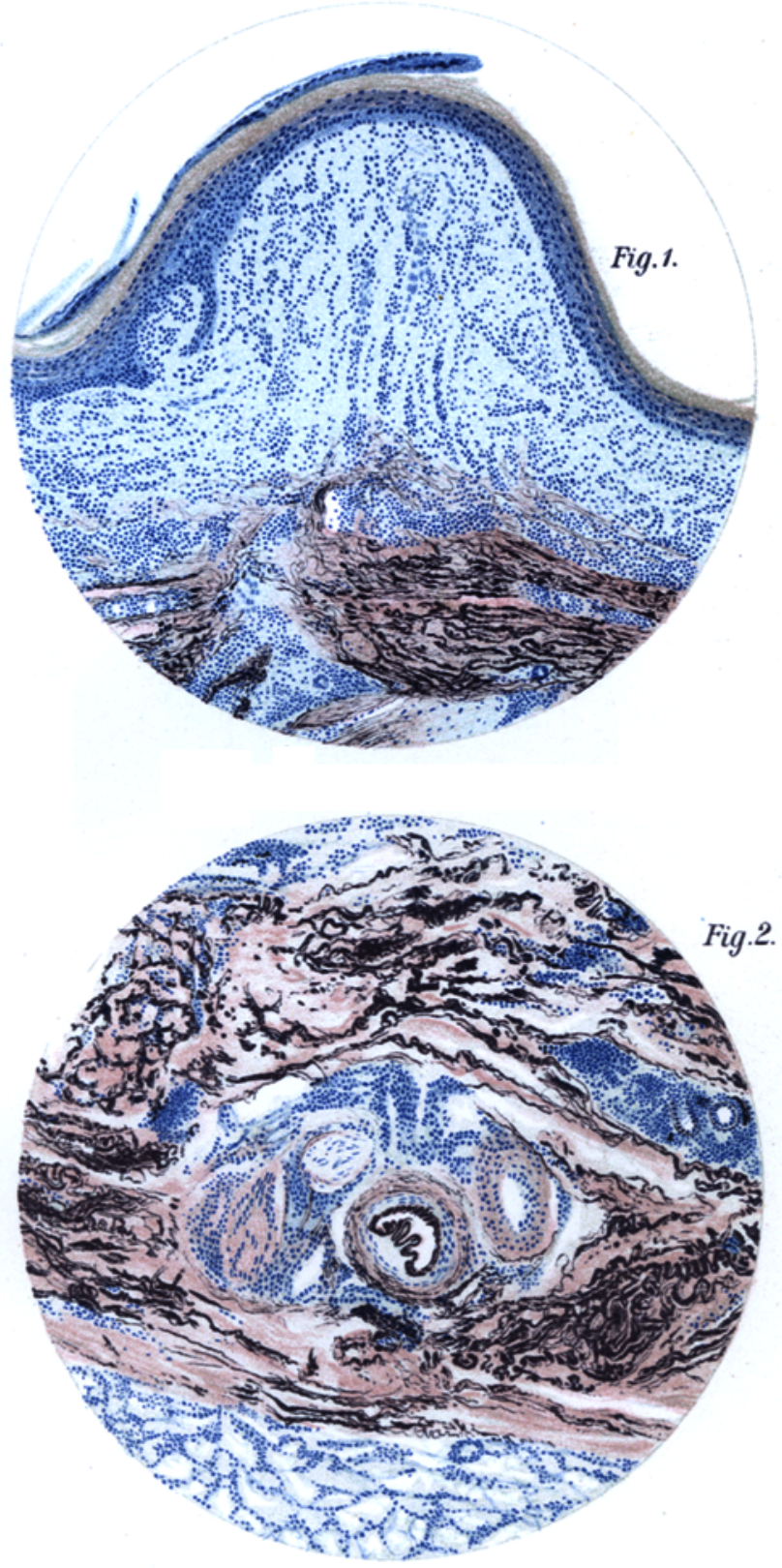

August Halle: Über einen Fall von Pityriasis rubra (Hebra) 